\title{
Differences in Event-Related Potential Responses to Japanese Onomatopoeias and Common Words
}

\author{
Yuka Egashira1 ${ }^{*}$, Damee Choi ${ }^{2,3}$, Midori Motoi ${ }^{3}$, Takayuki Nishimura4 \\ Shigeki Watanuki ${ }^{3}$ \\ ${ }^{1}$ Department of Kansei Science, Graduate School of Integrated Frontier Sciences, Kyushu University, \\ Fukuoka, Japan \\ ${ }^{2}$ Research Fellow of the Japan Society for the Promotion of Science, Tokyo, Japan \\ ${ }^{3}$ Faculty of Design, Kyushu University, Fukuoka, Japan \\ ${ }^{4}$ Department of Public Health, Nagasaki University Graduate School of Biomedical Sciences, Nagasaki, Japan \\ Email: *yu-kaegashira@kyudai.jp
}

Received 28 August 2015; accepted 16 October 2015; published 19 October 2015

Copyright (C) 2015 by authors and Scientific Research Publishing Inc.

This work is licensed under the Creative Commons Attribution International License (CC BY). http://creativecommons.org/licenses/by/4.0/

(c) $\underset{\mathrm{EY}}{\mathrm{B}}$ Open Access

\begin{abstract}
In this study, we measured event-related potential (ERP) responses to onomatopoeias (imitative words and mimetic words) and common words. Previous studies have shown that onomatopoeias are cognitively processed differently than common words. However, whether the temporal aspects of cognitive processing differ between onomatopoeias and common words remains unclear. The amplitude of the late positive complex (LPC), an index of sustained cognitive processing, of the ERP response to onomatopoeias was smaller than that for common words. In addition, the difference in the amplitude of the LPC between onomatopoeias and common words appeared from 200 ms until $900 \mathrm{~ms}$ after stimulus onset, suggesting that onomatopoeias do not require obligatory attentional capture or continued processing and encoding. Furthermore, marked differences between onomatopoeias and common words were evident in the early stages of cognitive processing. These results suggest that the temporal aspects of cognitive processing differ between onomatopoeias and common words.
\end{abstract}

\section{Keywords}

Onomatopoeia, Cognitive Processing, Event-Related Potential, Late Positive Complex

\footnotetext{
${ }^{*}$ Corresponding author.
} 


\section{Introduction}

One of the characteristics of the Japanese language is that it contains numerous imitative words and mimetic words known as onomatopoeias (Asaga et al., 2008). Many Japanese onomatopoeias verbalize visual and cutaneous sensations (e.g., damp, wet air and gloomy feelings can both be described by the same onomatopoeia, namely "jime-jime”). Onomatopoeias have a unique word form in that they contain two meaningless and often identical vocal sounds (e.g., “zigzag” and "ticktack” in English, “giza-giza” and "kachi-kachi” in Japanese) (Iwasaki et al., 2007). Previous studies have elucidated the differences between onomatopoeias and common words. In their functional magnetic resonance imaging (fMRI) study, Hashimoto et al. (2006) reported that the cognitive processing of onomatopoeias involves regions of the brain not only for language processing, but also for environmental sound processing. However, to the best of our knowledge, no previous studies have investigated whether the temporal aspects of cognitive processing differ between onomatopoeias and common words when heard.

An event-related potential (ERP) is a kind of physiological index for measuring the cognitive process of stimuli. ERP characteristics vary with different stimuli; they can reflect cognitive processing of stimuli within $1000 \mathrm{~ms}$ after stimulus onset, and have excellent temporal resolution. Accordingly, we can investigate the difference in cognitive processing between onomatopoeias and common words employing a time aspect. The duration of cognitive processing is reflected by the late positive ERP component, which is elicited approximately $300 \mathrm{~ms}$ after stimulus onset. These positive ERP amplitudes appear clearly at the centro-parietal region and are generally larger for stimuli that require a high cognitive processing load (Kramer et al., 1985; Polich, 2007). Therefore, we define the ERP component elicited about $300 \mathrm{~ms}$ after stimulus onset as the late positive complex (LPC), and divide it into three time windows: early, middle, and late.

Previous studies using ERP have shown that the human voice elicits a larger LPC than acoustic or environmental sounds (Clarke et al., 2000; Levy et al., 2003). As onomatopoeias are mimetic words, and the region of the brain involved in the cognitive processing of onomatopoeias is the same as that involved in the cognitive processing of sounds, onomatopoeias are considered similar to sounds. We hypothesized that the LPC evoked in response to onomatopoeias would be smaller than that for common words. The purpose of this study was to investigate for and clarify any existing differences in the LPC between onomatopoeias and common words.

Furthermore, it is well-known that the physical characteristics of auditory stimuli (e.g., loudness, frequency, and pitch) affect ERP waveforms. These effects can be reflected by the N1 component, which is an automatic response to the perception of stimuli. The peak of this component occurs about 50 - 150 ms after stimulus onset, and is observed over the fronto-central region (Näätänen \& Picton, 1987). If significant differences are seen in the N1 between onomatopoeias and common words, the effects of the physical characteristics of auditory stimuli on LPC amplitude must be taken into account. Therefore, in addition to the LPC, we also focused on the N1.

\section{Methods}

\subsection{Participants}

This study comprised a total of 20 male university and graduate students (mean age \pm standard deviation, $23 \pm$ 1.06 years). All participants were native Japanese speakers, healthy, and right-handed. This study was approved by the ethics committee of the Kyushu University Faculty of Design, and informed consent was obtained from all participants before the study began.

\subsection{Auditory Stimuli}

We chose a total of eight words (Table 1). Because onomatopoeias are based on and attempt to describe tactile, auditory, and visual sensations, it is difficult to find common words that convey identical meanings. For example, "gira-gira," an onomatopoeia that refers to glaring sunlight and desires, has no one-word equivalent in the common vocabulary. Therefore, for comparison, we selected onomatopoeias and common words for "dry" and "humid," which do have identical meanings.

For the auditory stimuli, we recorded speech spoken by a native Japanese female speaker. We used a female voice in an attempt to maintain the arousal level of the participants (Sokhi et al., 2005; Jones et al., 2010). The maximum sound pressure level was adjusted to $75 \pm 1 \mathrm{~dB}(\mathrm{~A})$. All auditory stimuli were presented through headphones. In addition, the participants assessed all auditory stimuli as pleasant or unpleasant on a scale from -100 to 100 (unpleasant: -100, neutral: 0, pleasant: 100). 
Table 1. List of auditory stimuli.

\begin{tabular}{|c|c|c|c|}
\hline Meaning & Words (pronunciation) & Duration of stimuli (ms) & Mean (SD) number of trials for ERP waveforms \\
\hline \multirow{6}{*}{ Dry } & Onor & poeias & \multirow{4}{*}{$52.0(18.9)$} \\
\hline & Sara-sara & 770 & \\
\hline & Kara-kara & 707 & \\
\hline & \multicolumn{2}{|c|}{ Common words } & \\
\hline & Kawaita & 772 & \multirow{2}{*}{$51.8(19.4)$} \\
\hline & Kansoushita & 922 & \\
\hline \multicolumn{3}{|c|}{ Onomatopoeias } & \multirow{4}{*}{$52.8(20.2)$} \\
\hline \multirow{5}{*}{ Humid } & Jime-jime & 679 & \\
\hline & Jito-jito & 770 & \\
\hline & \multicolumn{2}{|c|}{ Common words } & \\
\hline & Shimetta & 791 & \multirow{2}{*}{$53.3(19.8)$} \\
\hline & Shiketta & 778 & \\
\hline
\end{tabular}

SD: standard deviation.

\subsection{Procedures}

We measured ERPs while the participants listened to the auditory stimuli in a soundproof, electromagnetically-shielded, climate-controlled room (air temperature, $28^{\circ} \mathrm{C} \pm 0.5^{\circ} \mathrm{C}$; relative humidity, $50 \% \pm 5 \%$ ). The background noise level was constant at $55 \mathrm{~dB}(\mathrm{~A})$. In order to prevent habituation, each auditory stimulus (Table 1) was played both normally and backwards. To maintain their arousal level, the participants performed dummy button-pressing tasks in response to tone presentation (100 dB(A), $4000 \mathrm{~Hz}$ ). Each auditory stimulus and tone was presented 60 times, and all of the auditory stimuli were presented randomly. The ERP response for tone was not included in the analysis. The participant's button-pressing responses were monitored. None of the participants had a percentage of correct answers below $98 \%$.

\subsection{ERP Measurements and Analysis}

Electroencephalograms (EEGs) were recorded from 64 sites in accordance with the international 10 - 20 system using an EEG cap (HydroCel Geodesic Sensor Net, Electrical Geodesics, Inc., Eugene, OR, USA), amplified, and measured (Net Amps 200 64-channel EEG Amplifier, Net Station version 4.1.2, Electrical Geodesics, Inc.). Electrode resistance was maintained at $\leq 100 \mathrm{k} \Omega$ throughout the experiment, and signals were continuously recorded with a sampling frequency of $500 \mathrm{~Hz}$ using a 0.1 - $100 \mathrm{~Hz}$ band pass hardware filter. We used the online $\mathrm{Cz}$ reference and recalculated off-line relative to the average signal from all electrodes as the reference. EMSE Data Editor (version 5.2; Source Signal Imaging Inc., San Diego, CA, USA) was used for analysis (band pass software filter: 0.5 - $30 \mathrm{~Hz}$ ). Trials including artifacts above $\pm 60 \mu \mathrm{V}$ were manually rejected. A -300 to $1500 \mathrm{~ms}$ range was averaged to obtain ERP waveforms (baseline: -300 to $0 \mathrm{~ms}$ ). ERP waveforms for words with identical type and meaning were averaged. As a result, we derived the following four ERP waveforms: onomatopoeias meaning "dry”; onomatopoeias meaning "humid”; common words meaning "dry"; and common words meaning "humid”. In addition, we calculated ERP waveforms for onomatopoeias or common words played backwards averaged by type.

We then calculated the mean amplitudes for the following time windows: 50 - 150 ms (N1); 200 - 500 ms (early LPC); 500 - 900 ms (middle LPC); and 900 - 1500 ms (late LPC). LPC time windows were further divided based on previous studies (Hajcak et al., 2009) and our derived ERP waveforms. The averaged waveforms over the fronto-central region (FC1, FCz, and FC2) were analyzed for the N1, and the waveforms over the occipital posterior region (Pz, POz, P1 and P2) were analyzed for the three LPCs. 


\subsection{Statistical Analysis}

For N1 and LPC data, we conducted two-way repeated-measure analysis of variance using SPSS Statistics (Version 20; SPSS, Chicago, IL, USA) with word type (onomatopoeias vs common words) and meaning (“dry” vs "humid") as dependent variables. If a significant main effect was found, multiple comparisons were performed using the Bonferroni method. To confirm the effect of onomatopoeia repetition, we performed a paired t-test for the LPC s of backwards stimuli (onomatopoeias vs common words). In addition, to investigate the association between LPC $s$ and emotional value, we performed Pearson correlation analysis to compare LPC $s$ and the emotional value of auditory stimuli. All statistical results were considered significant at $p<0.05$.

\section{Results}

The grand mean waveforms of the fronto-central and occipital posterior regions are shown in Figure 1. No significant main or interaction effects were found between word type and meaning for $\mathrm{N} 1$ (word type: $\mathrm{F}(1,19)=$ $0.25, p=0.619, \eta^{2} \mathrm{p}=0.01$; word meaning: $\mathrm{F}(1,19)=4.27, p=0.052, \eta^{2} \mathrm{p}=0.18$; word type and meaning: $\left.\mathrm{F}(1,19)=0.42, p=0.523, \eta^{2} \mathrm{p}=0.00\right)$.

For early and middle LPCs (Figure 2), a significant main effect was found for word type (early LPC: $\mathrm{F}(1,19)$ $=7.13, p<0.05, \eta^{2} \mathrm{p}=0.27$; middle LPC: $\left.\mathrm{F}(1,19)=9.26, p<0.01, \eta^{2} \mathrm{p}=0.32\right)$. Subtest results showed that each LPC amplitude was smaller during processing of onomatopoeias than during processing of common words. No significant main effects were observed for word meaning (early LPC: $\mathrm{F}(1,19)=0.002, p=0.96, \eta^{2} \mathrm{p}=0.00$; middle LPC: $\mathrm{F}(1,19)=0.013, p=0.90, \eta^{2} \mathrm{p}=0.01$ ) or for interactions between word type and meaning (early
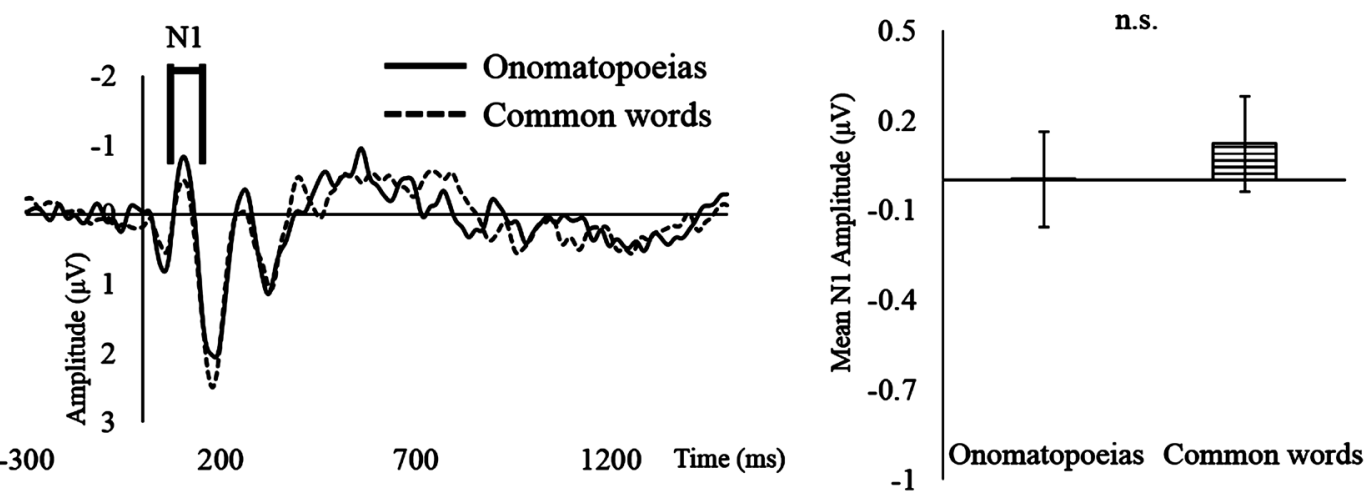

Figure 1. Grand mean event-related potential (ERP) waveforms over the fronto-central region (left) and the mean N1 amplitudes (right). No significant difference was found between onomatopoeias and common words in the mean N1.
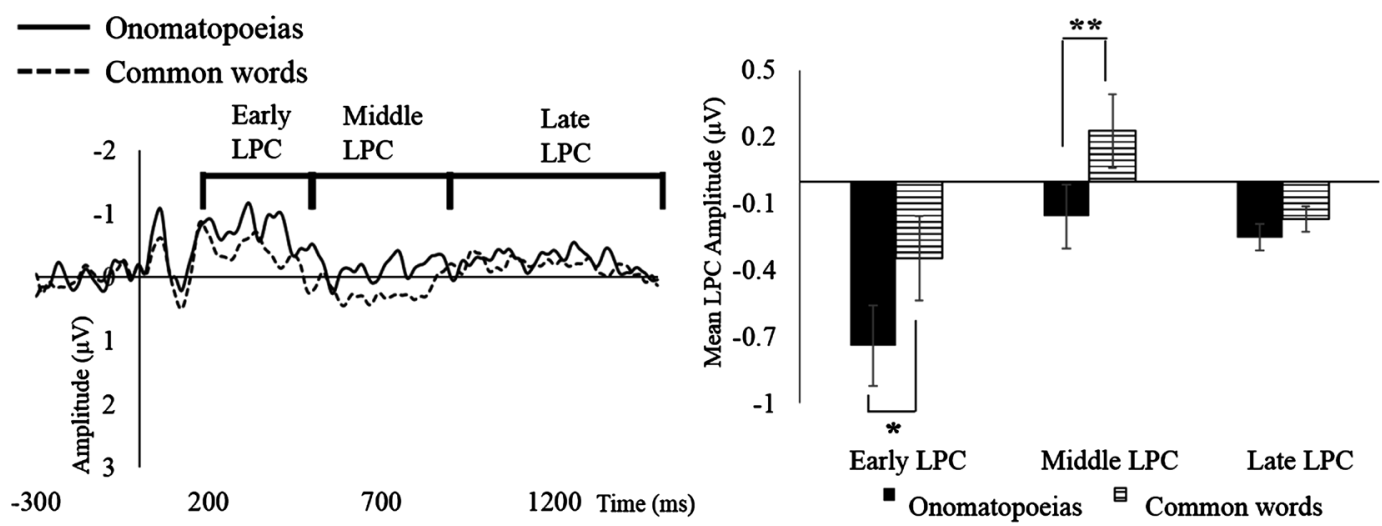

Figure 2. Grand mean event-related potential (ERP) waveforms over the over the occipital posterior region (left) and the mean LPC amplitudes (right). The early and middle LPCs were significantly smaller for onomatopoeias than for common words. ${ }^{*} p<0.05,{ }^{* *} p<0.01$ by the Bonferroni method. LPC: late positive complex. 
LPC: $\mathrm{F}(1,19)=0.37 ; p=0.54, \eta^{2} \mathrm{p}=0.01$; middle LPC: $\left.\mathrm{F}(1,19)=0.32, p=0.57, \eta^{2} \mathrm{p}=0.01\right)$. For late LPC, no significant main effects or interactions between word type and meaning were observed. In addition, on topographic maps of LPC amplitudes (onomatopoeias - common words) early and middle LPC amplitudes for onomatopoeias were more negative than those for common words (Figure 3).

In consideration of stimuli duration, we also performed the same tests without using the longest common word for dry ("kansoushita"), obtaining similar results. In addition, a significant main effect for word type was found for early and middle LPCs (early LPC: $\mathrm{F}(1,19)=4.729, p<0.05, \eta^{2} \mathrm{p}=0.19$, onomatopoeias $<$ common words; middle LPC: $\mathrm{F}(1,19)=10.635, p<0.005, \eta^{2} \mathrm{p}=0.35$, onomatopoeias < common words; late LPC: $\mathrm{F}(1$, $19)=2.392, p=0.13, \eta^{2} \mathrm{p}=0.11$ ). In the backwards LPC analysis, the middle LPC amplitude was significantly larger during processing of onomatopoeias than during processing of common words $(\mathrm{t}=2.46, \mathrm{df}=19, p<0.05$, $\eta^{2}=0.24$ ). No significant differences were found in early and late LPCs between onomatopoeias and common words (early LPC: $\mathrm{t}=-0.55, \mathrm{df}=19, p=0.57, \eta^{2}=0.01$; late LPC: $\mathrm{t}=0.44, \mathrm{df}=19, p=0.44, \eta^{2}=0.01$ ).

The mean value of subjective evaluation tests for auditory stimuli and results from the correlation analysis between the three LPCs and the emotional value of auditory stimuli are shown in Table 2. No significant relationships were found between the three LPCs and the emotional scores (Table 2).

\section{Discussion}

In this study, in order to investigate differences in the temporal aspects of cognitive processing, we obtained ERP responses to onomatopoeias and common words that were heard by the study participants. First, we confirmed that there were no differences in the N1, which reflects perceptual differences between auditory stimuli, between onomatopoeias and common words. High-intensity stimuli result in a larger N1 amplitude than lowintensity stimuli (Näätänen \& Picton, 1987). Based on our results, no significant difference was evident in the N1. Therefore, the LPC was not affected by preferential early attentional capture of the physical characteristics of the auditory stimuli.

Although the early and middle LPCs were significantly smaller for onomatopoeias than for common words, no significant differences were seen in the late LPC. High-arousal stimuli have been shown to elicit more positive LPC amplitudes than low-arousal stimuli (Hinojosa et al., 2012); these more positive amplitudes indicate sustained attention to stimuli (Weinberg \& Hajcak, 2011). Accordingly, recent results have shown that the arousal level for onomatopoeias is lower than that for common words, and that participants do not pay as much attention to onomatopoeias as they do to common words. In addition, LPC time windows can reportedly be divided according to stage of cognitive processing (Hajcak et al., 2009). Some previous studies have also suggested that early, middle, and late LPCs reflect obligatory attentional capture (Halgren et al., 1998), continued processing and encoding (Knight \& Scabini, 1998), and sustained attention for stimuli reanalysis (Gable \& Adams, 2013), respectively. The present results indicate that the early and middle LPC amplitudes for onomatopoeias are relatively small. This suggests that onomatopoeias do not require obligatory attentional capture or continued processing and encoding.
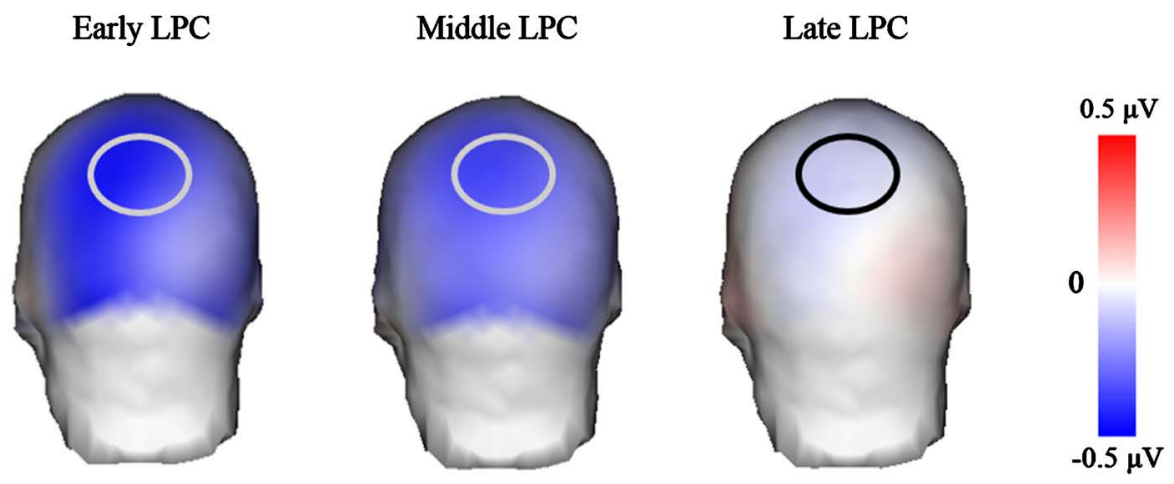

Figure 3. LPC topographic map (onomatopoeias—common words). The occipital posterior region is enclosed within a circle. Topographic maps of the early and middle LPC time windows show that onomatopoeias elicited smaller LPCs than common words over the occipital posterior region. LPC: late positive complex. 
Table 2. The mean emotional value of auditory stimuli and correlations between LPCs and the emotional value of auditory stimuli.

\begin{tabular}{|c|c|c|c|c|c|}
\hline \multirow{2}{*}{ Meaning } & \multirow{2}{*}{ Words (pronunciation) } & \multirow{2}{*}{ Emotional value (SD) } & \multicolumn{3}{|c|}{ Pearson's correlation coefficient (p-value) } \\
\hline & & & Early LPC & Middle LPC & Late LPC \\
\hline \multirow{6}{*}{ Dry } & Onomatopoeias & & & & \\
\hline & Sara-sara & $54.30(6.45)$ & $0.26(0.25)$ & $-0.06(0.80)$ & $0.33(0.15)$ \\
\hline & Kara-kara & $-11.05(8.33)$ & $-0.09(0.68)$ & $-0.22(0.33)$ & $-0.12(0.60)$ \\
\hline & Common words & & & & \\
\hline & Kawaita & $8.35(8.07)$ & $0.03(0.88)$ & $-0.06(0.80)$ & $0.03(0.87)$ \\
\hline & Kansoushita & $-16.20(8.51)$ & $0.10(0.67)$ & $0.06(0.78)$ & $0.17(0.46)$ \\
\hline \multirow{6}{*}{ Humid } & Onomatopoeias & & & & \\
\hline & Jime-jime & $-62.40(4.19)$ & $-0.21(0.36)$ & $-0.00(0.99)$ & $-0.23(0.32)$ \\
\hline & Jito-jito & $-65.85(4.36)$ & $0.00(0.98)$ & $0.00(0.99)$ & $0.03(0.89)$ \\
\hline & Common words & & & & \\
\hline & Shimetta & $-43.10(5.06)$ & $-0.37(0.10)$ & $-0.22(0.34)$ & $-0.39(0.08)$ \\
\hline & Shiketta & $-46.10(6.55)$ & $0.09(0.68)$ & $0.08(0.72)$ & $-0.00(0.97)$ \\
\hline
\end{tabular}

SD: standard deviation, LPC: late positive complex.

However, no significant difference was found in the late LPC between onomatopoeias and common words. According to a previous study (Friederici, 2002), speech perceptual processing continues until about $1000 \mathrm{~ms}$ after stimuli onset. In this study, no significant differences were found between onomatopoeias and common words within 900 - 1500 ms, since the cognitive processing of auditory stimuli was considered complete.

The smaller LPC associated with onomatopoeias may have been due to a variety of reasons other than word characteristics, such as the emotional value and physical characteristics of auditory stimuli (stimuli duration, sound repetition). No significant relationship was found between emotional value and LPC amplitudes using Pearson correlation analysis (Table 2); this suggests that LPC amplitudes were not affected by emotional value. The early and middle LPCs were significantly smaller for onomatopoeias than for common words, but no significant difference was found for the late LPC, even when excluding the longest auditory stimulus. These results suggest that LPC amplitudes are not affected by the duration of auditory stimuli. In addition, because onomatopoeias are often composed of two identical sounds, while common words are typically composed of different sounds, we examined backwards LPCs. Even when auditory stimuli were played backwards, no changes were seen in the sound characteristics of onomatopoeias. As a result, no significant differences were found in the early and late LPCs between onomatopoeias and common words. However, middle LPCs were significantly larger for onomatopoeias than for common words; this result was opposite from that observed when playing sounds normally. Accordingly, auditory stimuli composed of two identical sounds elicited larger middle LPCs than auditory stimuli composed of different sounds. Therefore, when played normally, the sound characteristics of onomatopoeias and common words did not appear to affect the early and middle LPCs. Based on these considerations, the smaller early and middle LPCs elicited by onomatopoeias appear to be result of differences in physical characteristics between onomatopoeias and common words.

One reason that onomatopoeias elicit smaller early and middle LPCs could be differences in the word image for onomatopoeias and common words. Lupyan \& Thompson-Schill (2012) reported that common words activate conceptual information more effectively than onomatopoeias. In addition, Boutonnet \& Lupyan (2015) reported that common words guide early visual processing more rapidly than equally informative nonverbal sounds such as a dog bark. Those findings suggest that common words evoke stronger conceptual information than onomatopoeias. Without context, common words that evoke stronger conceptual images than their onomatopoeia counterparts would be assessed as being more informative and useful. Another study (Ritter et al., 1988) found that stimuli containing a large amount of information elicited a large early LPC. Moreover, for adults, 
onomatopoeias may be assessed as less important than common words because onomatopoeias are not generally used in formal situations. For these reasons, common words tend to more frequently require obligatory attentional capture and continued processing and encoding compared with onomatopoeias.

In addition, the cognitive processing of onomatopoeias varies from that of common words. Hashimoto et al. (2006) measured responses to animal cries, the human voice pronouncing animal names, and onomatopoeic cries using fMRI. They found that onomatopoeias activated brain regions related to the recognition of animal cries and human pronunciation of animal names. In addition, the right superior temporal sulcus (STS) was more strongly activated by onomatopoeias than by human pronunciation of animal names. Kanero et al. (2014) also reported that onomatopoeias activate the right STS. The right STS is associated with environmental sound processing (Thierry et al., 2003); therefore, although onomatopoeias are speech, they are processed similarly to environmental sounds. Previous studies on ERPs and fMRI have shown that the arousal level for speech sounds is higher than that for environmental sounds (Belin et al., 2004), and that speech sounds lead to more attentional capture than environmental sounds (Levy et al., 2003). In this study, onomatopoeias elicited smaller LPCs because of their similarity to environmental sounds.

On the other hand, our results conflict with those reported by Lockwood \& Tuomainen (2015) in their investigation of cognitive differences between onomatopoeias and common words in sentences. A large LPC (400 $800 \mathrm{~ms}$ time window) was elicited by onomatopoeias, which they suggested may reflect facilitated lexical retrieval of common words compared with onomatopoeias. The difference between their results and those from the present study could be explained by the context effect. Lockwood \& Tuomainen (2015) used sentences as the stimuli; in contrast, we used words with no context. When listening only, onomatopoeias required a smaller LPC than common words.

This study did have a few limitations. First, we only used auditory stimuli. If we had used visual stimuli for onomatopoeias, repetitive characters would have been seen, thereby eliciting a smaller LPC than common words. Therefore, it is possible that a specific modality can influence these results. Second, a previous study (Schupp et al., 2006) reported that emotional stimuli elicit large LPCs. However, in this study, we used non-emotional stimuli. Our results showed that LPCs for onomatopoeias were smaller than those for common words. If we had used affective words as stimuli, affective onomatopoeias might have yielded smaller LPCs than affective common words. However, in Japanese, onomatopoeias provide vivid descriptions of a speaker's senses and impressions. Therefore, it is possible that affective onomatopoeias would have required a larger LPC. Differences between onomatopoeias and common words should be examined in more detail in future studies.

\section{Conclusion}

In this study, we focused on the differences in the passive auditory cognitive process between onomatopoeias and common words using ERPs, which have excellent temporal resolution. Onomatopoeias elicited smaller LPCs than common words, as stimuli that require obligatory attentional capture or continued processing and encoding elicit more positive LPC amplitudes. Therefore, these results show that onomatopoeias require less attentional capture than common words. In addition, although differences were observed in the cognitive processing of onomatopoeias and common words in the early to the middle LPCs, no significant differences were seen in the late LPC. This study provides new evidence that the temporal aspects of auditory cognitive processing differ between onomatopoeias and common words.

\section{Acknowledgements}

We would like to offer our special thanks to Dr. Gerard B. REMIJN, Associate Professor of Kyushu University, for proofreading this article. We also thank Dr. Michiko ASANO, Assistant Professor of Rikkyo University, for her helpful advice and comments for this article.

\section{References}

Asaga, C., Mukarramah, Y., \& Watanabe, C. (2008). ONOMATOPEDIA: Onomatopoeia Online Example Dictionary System Extracted from Data on the Web. Progress in WWW Research and Development (pp. 601-612). Berlin: Springer. http://dx.doi.org/10.1007/978-3-540-78849-2_60

Belin, P., Fecteau, S., \& Bedard, C. (2004). Thinking the Voice: Neural Correlates of Voice Perception. Trends in Cognitive Sciences, 8, 129-135. http://dx.doi.org/10.1016/j.tics.2004.01.008 
Boutonnet, B., \& Lupyan, G. (2015). Words Jump-Start Vision: A Label Advantage in Object Recognition. The Journal of Neuroscience, 35, 9329-9335. http://dx.doi.org/10.1523/JNEUROSCI.5111-14.2015

Clarke, S., Bellmann, A., Meuli, R. A., Assal, G., \& Steck, A. J. (2000). Auditory Agnosia and Auditory Spatial Deficits Following Left Hemispheric Lesions: Evidence for Distinct Processing Pathways. Neuropsychologia, 38, 797-807. http://dx.doi.org/10.1016/S0028-3932(99)00141-4

Friederici, A. D. (2002). Towards a Neural Basis of Auditory Sentence Processing. Trends in Cognitive Sciences, 6, 78-84. http://dx.doi.org/10.1016/S1364-6613(00)01839-8

Gable, P. A., \& Adams, D. L. (2013). Nonaffective Motivation Modulates the Sustained LPP (1,000-2,000 ms). Psychophysiology, 50, 1251-1254. http://dx.doi.org/10.1111/psyp.12135

Hajcak, G., Dunning, J. P., \& Foti, D. (2009). Motivated and Controlled Attention to Emotion: Time-Course of the Late Positive Potential. Clinical Neurophysiology, 120, 505-510. http://dx.doi.org/10.1016/j.clinph.2008.11.028

Halgren, E., Marinkovic, K., \& Chauvel, P. (1998). Generators of the Late Cognitive Potentials in Auditory and Visual Oddball Tasks. Electroencephalography and Clinical Neurophysiology, 106, 156-164. http://dx.doi.org/10.1016/S0013-4694(97)00119-3

Hashimoto, T., Usui, N., Taira, M., Nose, I., Haji, T., \& Kojima, S. (2006). The Neural Mechanism Associated with the Processing of Onomatopoeic Sounds. Neuroimage, 31, 1762-1770. http://dx.doi.org/10.1016/j.neuroimage.2006.02.019

Hinojosa, J. A., Méndez-Bértolo, C., \& Pozo, M. A. (2012). High Arousal Words Influence Subsequent Processing of Neutral Information: Evidence from Event-Related Potentials. International Journal of Psychophysiology, 86, $143-151$. http://dx.doi.org/10.1016/j.ijpsycho.2012.06.001

Iwasaki, N., Vinson, D. P., \& Vigliocco, G. (2007). What Do English Speakers Know about Gera-Gera and Yota-Yota?: A Cross-Linguistic Investigation of Mimetic Words for Laughing and Walking. Japanese-Language Education around the Globe, 17, 53-78.

Jones, B. C., Feinberg, D. R., DeBruine, L. M., Little, A. C., \& Vukovic, J. (2010). A Domain-Specific Opposite-Sex Bias in Human Preferences for Manipulated Voice Pitch. Animal Behaviour, 79, 57-62. http://dx.doi.org/10.1016/j.anbehav.2009.10.003

Kanero, J., Imai, M., Okuda, J., Okada, H., \& Matsuda, T. (2014). How Sound Symbolism Is Processed in the Brain: A Study on Japanese Mimetic Words. PLoS ONE, 9, e97905. http://dx.doi.org/10.1371/journal.pone.0097905

Knight, R. T., \& Scabini, D. (1998). Anatomic Bases of Event-Related Potentials and Their Relationship to Novelty Detection in Humans. Journal of Clinical Neurophysiology, 15, 3-13. http://dx.doi.org/10.1097/00004691-199801000-00003

Kramer, A. F., Wickens, C. D., \& Donchin, E. (1985). Processing of Stimulus Properties: Evidence for Dual-Task Integrality. Journal of Experimental Psychology: Human Perception and Performance, 11, 393-408. http://dx.doi.org/10.1037/0096-1523.11.4.393

Levy, D. A., Granot, R., \& Bentin, S. (2003). Neural Sensitivity to Human Voices: ERP Evidence of Task and Attentional Influences. Psychophysiology, 40, 291-305. http://dx.doi.org/10.1111/1469-8986.00031

Lockwood, G., \& Tuomainen, J. (2015). Ideophonesin Japanese Modulate the P2 and Late Positive Complex Responses. Frontiers in Psychology, 6, 933. http://dx.doi.org/10.3389/fpsyg.2015.00933

Lupyan, G., \& Thompson-Schill, S. L. (2012). The Evocative Power of Words: Activation of Concepts by Verbal and Nonverbal Means. Journal of Experimental Psychology: General, 141, 170-186. http://dx.doi.org/10.1037/a0024904

Näätänen, R., \& Picton, T. (1987). The N1 Wave of the Human Electric and Magnetic Response to Sound: A Review and an Analysis of the Component Structure. Psychophysiology, 24, 375-425.

http://dx.doi.org/10.1111/j.1469-8986.1987.tb00311.x

Polich, J. (2007). Updating P300: An Integrative Theory of P3a and P3b. Clinical Neurophysiology, 118, 2128-2148. http://dx.doi.org/10.1016/j.clinph.2007.04.019

Ritter, W., Simson, R., \& Vaughan, H. G. (1988). Effects of the Amount of Stimulus Information Processed on Negative Event-Related Potentials. Electroencephalography and Clinical Neurophysiology, 69, 244-258. http://dx.doi.org/10.1016/0013-4694(88)90133-2

Schupp, H. T., Flaisch, T., Stockburger, J., \& Junghöfer, M. (2006). Emotion and Attention: Event-Related Brain Potential Studies. Progress in Brain Research, 156, 31-51. http://dx.doi.org/10.1016/S0079-6123(06)56002-9

Sokhi, D. S., Hunter, M. D., Wilkinson, I. D., \& Woodruff, P. W. (2005). Male and Female Voices Activate Distinct Regions in the Male Brain. Neuroimage, 27, 572-578. http://dx.doi.org/10.1016/j.neuroimage.2005.04.023

Thierry, G., Giraud, A. L., \& Price, C. (2003). Hemispheric Dissociation in Access to the Human Semantic System. Neuron, 38, 499-506. http://dx.doi.org/10.1016/S0896-6273(03)00199-5

Weinberg, A., \& Hajcak, G. (2011). The Late Positive Potential Predicts Subsequent Interference with Target Processing. Journal of Cognitive Neuroscience, 23, 2994-3007. http://dx.doi.org/10.1162/jocn.2011.21630 